\title{
Correlation between Dietary Intake and Inflammatory Biomarkers in a Tunisian Obese Group
}

\author{
Manel Ayoub' ${ }^{1}$, Chedia Zouaoui ${ }^{2}$, Nedra Grira1 ${ }^{1}$, Radhia Kochkar ${ }^{3}$, Nejla Stambouli1, \\ Chaker Bouguerra4 ${ }^{4}$, Borni Zidi², Ezzedine Ghazouani' ${ }^{3}$, Chakib Mazigh ${ }^{1}$, Zied Aouni1 ${ }^{*}$ \\ ${ }^{1}$ Biochemistry Department, Research Unit, Military Hospital of Tunis, Tunis, Tunisia \\ ${ }^{2}$ Endocrinology Department, Research Unit, Military Hospital of Tunis, Tunis, Tunisia \\ ${ }^{3}$ Immunology Department, Military Hospital of Tunis, Tunis, Tunisia \\ ${ }^{4}$ Epidemiology Department, Research Unit, Military Hospital of Tunis, Tunis, Tunisia \\ Email: *aouni_zied@yahoo.fr
}

Received 12 October 2015; accepted 20 November 2015; published 23 November 2015

Copyright (C) 2015 by authors and Scientific Research Publishing Inc.

This work is licensed under the Creative Commons Attribution International License (CC BY). http://creativecommons.org/licenses/by/4.0/

(c) (i) Open Access

\begin{abstract}
Aims: This study aims to determine the inflammatory status and evaluate the correlation between dietary intake and inflammatory biomarkers in a Tunisian obese group. Material and Methods: This is an open cross-sectional study that includes 81 individuals divided into two groups: a group of 42 obese patients recruited from the endocrinology department and a control group of 39 nonobese healthy subjects. All participants received a food survey, anthropometric measurements and blood sampling. Pro-inflammatory cytokines (IL-6, IL-8, TNF- $\alpha$ ), and hs-CRP were measured in patients and controls. Results: Dietary data revealed significant increases in food intake in obese patients compared to controls. The results of the laboratory parameters showed a statistically significant increase in IL-8, TNF- $\alpha$ and blood glucose in obese compared to controls. The analysis of correlations among the parameters studied showed that the plasma level of IL-6 was positively correlated with dietary fat intake $\left(r=0.70 ; p<10^{-3}\right)$ and carbohydrate $(r=0.54 ; p=0.03)$ and the dietary intake expressed in calories $\left(r=0.64 ; p<10^{-3}\right)$. Conclusion: dietary imbalances in obese were observed and characterized by an excessive intake of calories, fat and carbohydrates. The excessive carbohydrate and fat intake is associated with increased IL- 6 and TNF- $\alpha$ plasma levels.
\end{abstract}

\section{Keywords}

Obesity, Dietary Intake, Inflammatory Biomarkers

\footnotetext{
${ }^{*}$ Corresponding author.
}

How to cite this paper: Ayoub, M., Zouaoui, C., Grira, N., Kochkar, R., Stambouli, N., Bouguerra, C., Zidi, B., Ghazouani, E., Mazigh, C. and Aouni, Z. (2015) Correlation between Dietary Intake and Inflammatory Biomarkers in a Tunisian Obese Group. Journal of Biosciences and Medicines, 3, 108-113. http://dx.doi.org/10.4236/jbm.2015.311014 


\section{Introduction}

Obesity is the epidemic of the $21^{\text {st }}$ century. In developing countries, the number of obese people is increasing, and obesity is occurring at younger ages [1]. The fundamental cause of obesity and overweight is an energy imbalance between calories consumed and calories expended. As more is understood about obesity, the complexity of this chronic disorder becomes more apparent, exhibiting a multi-factorial etiology. Lifestyle factors such as diet and exercise continue to be recognized to play an important role in the development and progression of obesity and its comorbidities. However, genetic variation is also known to contribute to the obese phenotype [2]. The World Health Organization (WHO) has reported that obesity has been growing at an alarming rate, accounting for approximately $35 \%$ of the population. Obesity and overweight increase the risk of several serious chronic diseases, such as type 2 diabetes, cardiovascular disease, hypertension and stroke, hypercholesterolemia, and hypertriglyceridemia [3].

A common denominator that links these diseases to obesity is inflammation. While signals that initiate the inflammatory process remain unclear, emerging evidence suggests that nutrients and the modifications they cause in adipose tissue during situations of nutritional overload can activate immune sensors [4].

Adipose tissue, the main feature of obesity, was considered as an inert tissue mainly devoted to fat storage: However, it is now recognized as an active tissue in the regulation of physiological and pathological process, including immunity and inflammation. Adipose tissue produces and releases a variety of adipokines and cytokines, including leptin, adiponectin, TNF- $\alpha$, IL-6... [5]. Many studies have reviewed the role of diet and dietary factors on inflammation state and its relationship to obesity [6] [7].

In our study, we aimed to determine the inflammatory status and evaluate the correlation between dietary intake and inflammatory biomarkers in a Tunisian obese group.

\section{Materials and Methods}

This is a prospective open cross sectional study conducted at the Biochemistry department in collaboration with the Endocrinology Service in Military Hospital of Tunis.

\subsection{Study Population}

Our population consists of 80 subjects divided into two groups: a group of obese subjects consisted of 41 individuals admitted to the endocrinology department. Weight and height were measured on barefoot and lightly clothed subjects. Body mass index (BMI; $\mathrm{kg} / \mathrm{m}^{2}$ ) was calculated and obesity was defined as BMI $\geq 30 \mathrm{~kg} / \mathrm{m}^{2}$. And a control group containing 39 healthy individuals whose clinical and anthropometric measures have eliminated the diagnosis obesity. The study objectives and procedure were explained to all participants, before obtaining their consent to enroll into the study.

\subsection{Data Collection}

All patients and controls have received a complete physical examination with determination of anthropometric parameters (weight, height, waist circumference and hip circumference). The nutritional profile of obese subjects and controls was established through a food survey. They were asked to complete a week dietary record, and to report exhaustively the exact amount of foods and beverages ingested. The same dietitian calculated the total daily intake of calories, carbohydrates, lipids and proteins by BILNUT software using a food composition data base. The BILNUT method range from detailed individual weighed records collected over a variable period of time to food frequency questionnaires and dietary recalls.

\subsection{Blood Analyses}

The hs-CRP was performed by a particle enhanced immunonephelometry method using polystyrene particles coated with monoclonal antibodies specific to human CRP on the BN Pro Spec analyser (Siemens). The result is evaluated by comparison with a standard of known concentration. Proinflammatory cytokines (IL-6, IL-8 and TNF- $\alpha$ ) were measured by immunometric method with detection by chemiluminescence on Immulite system (Siemens). 


\subsection{Statistical Analysis}

Data were entered and analyzed by SPSS 19.0 software release. The results were expressed as the number of cases and percentages for categorical variables and mean and standard deviation for quantitative variables. The comparative study on independent series was performed using the $t$ test for quantitative variables and Chi-square test for categorical variables. The value of $\mathrm{p}=0.05$ is set as a threshold value below which a difference is taken as statistically significant.

\section{Results}

This study analyses a total of 81 subjects (42 obese patients and 39 non-obese healthy subjects).

Anthropometric and biochemical parameters for obese patients and controls are shown in Table 1.

The two study groups were matched for age and sex. It was noted that $92.7 \%$ of obese subjects had a family history of cardiovascular disease. Smoking was less common in obese compared to controls (17.1\% vs. 31.6\%), but without statistically significant difference.

Among the modifiable cardiovascular risk factors, hypertension was found in $36.6 \%$ of obese vs $5.4 \%$ of controls $\left(\mathrm{p}=10^{-3}\right)$. Dyslipidemia was observed in $14.6 \%$ of obese and $4.3 \%$ of controls. Similarly, $22 \%$ were diabetic obese compared with $4.9 \%$ of controls $(p=0.04)$.

The plasma levels of hs-CRP were significantly higher in obese $\left(\mathrm{p}<10^{-3}\right)$. We observed that the mean levels of TNF- $\alpha$ were significantly higher in obese compared to controls $(\mathrm{p}=0.028)$. Similarly, a significant increase of the mean values of IL- 8 was observed in the obese group $(p=0.013)$. The plasma levels of IL- 6 were higher in obese but this increase was not significant $(\mathrm{p}=0.3)$.

The daily intake of various nutrients is given in the Table 2.

Table 1. Anthropometric and biochemical parameters for obese patients and controls.

\begin{tabular}{cccc}
\hline & Obese $\mathbf{( n = 4 1 )}$ & Controls $(\mathbf{n}=\mathbf{3 9})$ & $\mathbf{p}$ \\
\hline Age & $43.44 \pm 10.34$ & $41.35 \pm 11.42$ & 0.21 \\
Gender M/W (\%) & $16.7 / 83.3$ & $17.9 / 82.1$ & NS \\
BMI & $39.17 \pm 7.35$ & $24.38 \pm 3.78$ & 0.015 \\
Tobacco (\%) & 17.1 & 31.6 & 0.001 \\
Hypertension (\%) & 36.6 & 5.4 & 0.018 \\
Dyslipidemia (\%) & 14.6 & 4.3 & 0.03 \\
Diabetes (\%) & 22 & 4.9 & $<10^{-3}$ \\
hs-CRP (mg/) & $5.46 \pm 4.8$ & $1.63 \pm 1.75$ & 0.028 \\
TNF $\boldsymbol{\alpha}$ (pg/ml) & $6.99 \pm 2.13$ & $6.00 \pm 1.60$ & 0.3 \\
IL-6 (pg/ml) & $2.78 \pm 1.85$ & $2.50 \pm 0.84$ & 0.013 \\
IL-8 (pg/ml) & $9.38 \pm 4.90$ & $6.73 \pm 2.37$ &
\end{tabular}

M/W: male/woman.

Table 2. The daily intake of various nutrients in obese and control subjects.

\begin{tabular}{cccc}
\hline & Obese $(\mathbf{n}=\mathbf{4 1})$ & Controls $(\mathbf{n}=\mathbf{3 9})$ & $\mathbf{p}$ \\
\hline Protides (g/24h) & $87.19 \pm 36.18$ & $55.9 \pm 14.9$ & $<10^{-3}$ \\
Carbohydrates (g/24h) & $298.5 \pm 184.99$ & $202.3 \pm 61.4$ & 0.1 \\
Lipids (g/24h) & $114.68 \pm 82.68$ & $70.5 \pm 19.6$ & 0.007 \\
Calories (Kcal/24h) & $2580 \pm 1494.15$ & $1704.4 \pm 374.9$ & 0.003 \\
\hline
\end{tabular}


The analysis of the association between dietary intake and pro-inflammatory cytokines in obese showed that the plasma levels of IL-6 was positively correlated with dietary fat intake $\left(r=0.70 ; p<10^{-3}\right)$ (Figure 1$)$ and carbohydrate $(\mathrm{r}=0.54 ; \mathrm{p}=0.03$ ) (Figure 2$)$ and the total dietary intake expressed in calories $\left(\mathrm{r}=0.64 ; \mathrm{p}<10^{-3}\right)$.

\section{Discussion}

Obesity is associated with a chronic increase of a set of inflammatory and lipid markers. Obese individuals in our series have high values of circulating TNF $\alpha$ and IL-8 compared to the control population. The mean values of IL-6 found in obese are higher than in the control population. However, this increase was not statistically significant. Our results are in agreement with most studies [8] [9].

During aggravation of obesity, there is an hypertrophy of the adipocytes. Indeed, adipose tissue of obese patients is the seat of macrophage accumulation [10]. These macrophages are a source of production of adipokines such as resistin and visfatin [11] and pro-inflammatory cytokines. In response to reservations of excess lipids, adipocytes secrete increasing amounts of inflammatory cytokines such as IL-6 and TNF- $\alpha$ and chemokines. These promote migration of macrophages in adipose tissue which increases the release of cytokines [12].

In our study, obese patients have significantly higher CRP concentrations compared to controls. Maria João Neuparth et al. [13] reported significantly higher CRP values in obese subjects compared to control groups. These authors have shown that CRP values tend to increase in overweight subjects. hs-CRP is produced by hepatocytes in response to proinflammatory cytokines, in particular IL-6, [14]. It should be noted that the liver and lymphoid organs are the main producers of these inflammatory mediators sites, but in obese individuals, fat

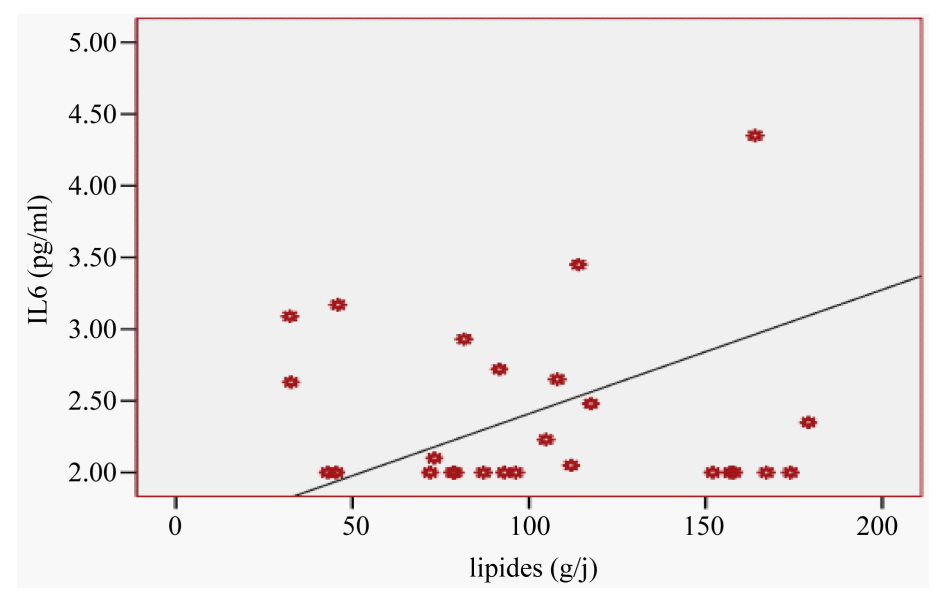

Figure 1. Correlation between lipids (g/j) and IL6 (pg/ml).

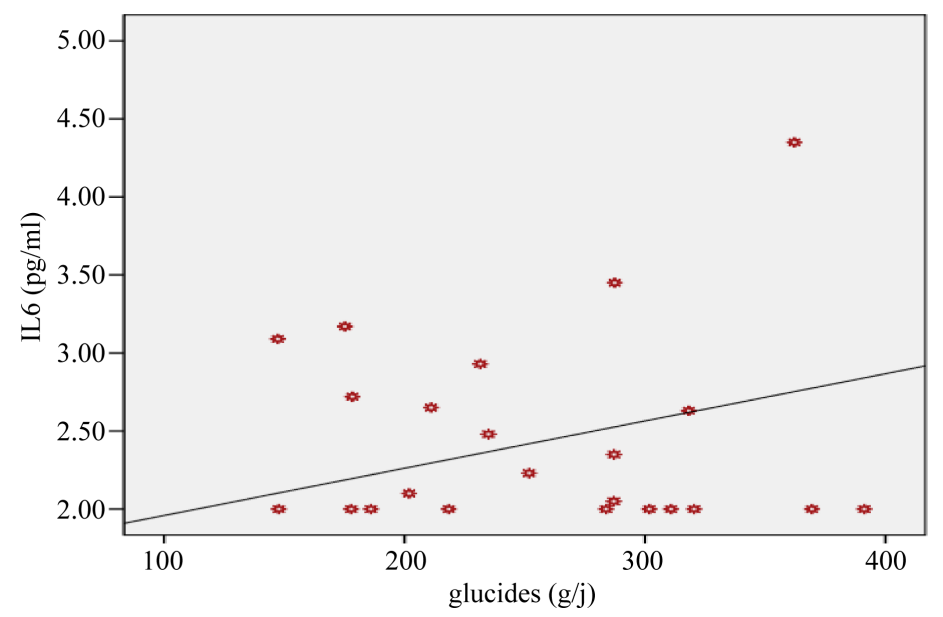

Figure 2. Correlation between glucides (g/j) and IL6 (pg/ml). 
tissue becomes a major producer of these markers [15]. The nutritional profile of obese established through food survey was characterized by excessive calorie intake means. Furthermore, the distribution of energy nutrients in daily calorie intake is unbalanced in favor of an excess of lipid and carbohydrate consumption. These results are comparable to those of Fennira et al. [16] in a study of 108 nearby Tunisian obese adolescents.

In our study, we found a statistically significant correlation between IL-6 and dietary carbohydrate intake on the one hand and the contributions by the other lipids.

Several studies have examined the effect of different dietary fatty acids on the production of IL-6 and expression of the gene of IL-6 into the cell. In vitro studies have reported that incubation of the adipocytes with palmitic acid increases expression of the gene and production of IL-6. Furthermore, in mice fed a diet rich in fatty acids the expression of IL-6 gene is three times higher than in mice fed a diet low in fatty acids [2].

Food is an important factor in regulating the immune response. Overeating leads to immuno-activation due to a susceptibility to an inflammatory disease. Therefore, optimal nutrition is necessary for a healthy immune balance. But, a nutrient overload causes obesity which is a chronic inflammatory condition [17].

Increasing dietary intake of fatty acids, especially in people who have an overweight or obese is associated with increased concentrations of inflammatory markers, inducing inflammatory changes in adipocytes and macrophages and increasing the gene expression and adipocytokine production. It has been shown that when adipocytes are exposed to dietary fatty acids such as palmitic acid, the production of IL-6 and expression of mRNA increases. Similarly monocytes were activated directly when exposed to dietary fatty acids [2].

The same modest reduction in weight improves the complications related to obesity. Many studies show that reducing food intake and increasing physical activity are factors that reduce inflammation. When loosing weight, a reduction of many molecules of inflammation is observed, it is established for CRP, IL-6, IL-8 and TNF- $\alpha$ [18].

\section{Conclusions}

We have highlighted the existence of an inflammatory state in obesity marked by an increase in TNF $\alpha$ levels, IL-8 and hs-CRP.

Nutritionally, dietary imbalances in obese were observed characterized by an excessive intake of calories, fat and carbohydrates. A dietary impact of these errors on the metabolic profile was noted. According to the results of our work, excessive carbohydrate and fat intake is associated with increased IL-6 and TNF alpha. the reduction of inputs and the correction of dietary errors are unavoidable in the management of obesity. In this context, we recommend, to prevent obesity, to avoid foods that are high in "energy density" or that have a lot of calories in a small amount of food and focus on low-calorie, nutrient-dense foods, such as fruits, vegetables and whole grains.

However, our study has some limitations. The most important is the relatively small size of our study population.

\section{Conflict of Interests}

The author had no conflict of interests to report.

\section{Acknowledgements}

The authors thank all the technicians of the biochemistry lab in military hospital of Tunis, especially Mr. Zeddini Imed and Mrs. Baccour Ilhem, for their contributions to the realization of this work.

\section{References}

[1] World Health Organization (2005) Fact Sheet: Obesity and Overweight. http://www.who.int/dietphysicalactivity/publications/facts/obesity/en/

[2] Joffe, Y.T., Collins, M. and Goedecke, J.H. (2013) The Relationship between Dietary Fatty Acids and Inflammatory Genes on the Obese Phenotype and Serum Lipids. Nutrients, 5, 1672-1705. http://dx.doi.org/10.3390/nu5051672

[3] World Health Organization (2008) Obesity [Internet]. WHO, Geneva. http://www.who.int/topics/obesity/en/

[4] Greenberg, A.S. and Obin, M.S. (2006) Obesity and the Role of Adipose Tissue in Inflammation and Metabolism. American Journal of Clinical Nutrition, 83, 461S-465S. 
[5] Galic, S., Oakhill, J.S. and Steinberg, G.R. (2010) Adipose Tissue as an Endocrine Organ. Molecular and Cellular Endocrinology, 316, 129-139. http://dx.doi.org/10.1016/j.mce.2009.08.018

[6] Galland, L. (2010) Diet and Inflammation. Nutrition in Clinical Practice, 10, 1164-1172. http://dx.doi.org/10.1177/0884533610385703

[7] Calder, P.C., Ahluwalia, N., Brouns, F., Buetler, T., Clement, K., Cunningham, K., et al (2011) Dietary Factors and Low Grade Inflammation in Relation to Overweight and Obesity. British Journal of Nutrition, 106, S5-S78. http://dx.doi.org/10.1017/s0007114511005460

[8] Khosravi, R., Ka, K., Huang, T., Khalili, S., Hong Nguyen, B., Nicolau, B. and Simon, D. (2013) Tumor Necrosis Factor- $\alpha$ and Interleukin-6: Potential Interorgan Inflammatory Mediators Contributing to Destructive Periodontal Disease in Obesity or Metabolic Syndrome. Mediators of Inflammation, 2013, 1-6. http://dx.doi.org/10.1155/2013/728987

[9] Agarwal, N., Chitrika, A., Bhattacharjee, J. and Jain, S.K. (2011) Correlation of Tumour Necrosis Factor- $\alpha$ and Interleukin-6 with Anthropometric Indices of Obesity and Parameters of Insulin Resistance in Healthy North Indian Population. JIACM, 12, 196-204.

[10] Pigeyre, M(2010). Évolution des concepts physiopathologiques de l’obésité. La Presse Médicale, 39, 907-912. http://dx.doi.org/10.1016/j.lpm.2010.05.015

[11] Schlienger, J.-L.(2010) Conséquences pathologiques de l’obésité. La Presse Médicale, 39, 913-920. http://dx.doi.org/10.1016/j.lpm.2010.04.018

[12] De Boer, M.D. (2013) Obesity, Systemic Inflammation, and Increased Risk for Cardiovascular Disease and Diabetes among Adolescents: A Need for Screening Tools to Target Interventions. Nutrition, 29, 379-386. http://dx.doi.org/10.1016/j.nut.2012.07.003

[13] Neuparth, M.J., Proença, J.B., Santos-Silva, A. and Coimbra, S. (2013) Adipokines, Oxidized Low-Density Lipoprotein, and C-Reactive Protein Levels in Lean, Overweight, and Obese Portuguese Patients with Type 2 Diabetes. ISRN obesity, 2013, Article ID: 142097. http://dx.doi.org/10.1155/2013/142097

[14] Kaur, J. (2014) A Comprehensive Review on Metabolic Syndrome. Cardiology Research and Practice, 2014, 1-22. http://dx.doi.org/10.1155/2014/281483

[15] Caldar, P.C., Ahluwalia, N., Brouns, F., Buetler, T., Clement, K. and Cunningham, K. (2011) Dietary Factors and Low-Grade Inflammation in Relation to Overweight and Obesity. BJN, 106, S1-S78. http://dx.doi.org/10.1017/s0007114511005460

[16] Fennira, E., Mahjoub, F., Abdesslem, H., Chaari, C., Gamoudi, A., Amrouche, C., et al. (2014) Alimentation de l'adolescent obèse tunisien: A propos de 108 cas. Nutrition Clinique et Métabolisme, 28, S155-S156. http://dx.doi.org/10.1016/s0985-0562(14)70809-x

[17] Lee, H., In, S. and Choue, R. (2013) Obesity, Inflammation and Diet. Pediatric Gastroenterology, Hepatology, and Nutrition, 3, 143-152. http://dx.doi.org/10.5223/pghn.2013.16.3.143

[18] Clément, K. and Vignes, S. (2009) Inflammation, adipokines et obésité. La Revue de Médecine Interne, 30, 824-832. http://dx.doi.org/10.1016/j.revmed.2009.03.363

\section{Abbreviations}

IL-6: Interleukin-6;

IL-8: Interleukin-8;

TNF- $\alpha$ : Tumour Necrosis Factor Alpha;

hsCRP: High Sensitivity C Reactive Protein. 